\title{
Evacuation System in a Building Using Cellular Automata for Pedestrian Dynamics
}

\author{
Muarifin1, Tri Harsono², Aliridho Barakbah² \\ Postgraduate of Information Engineering and Computer, Electronics Engineering \\ Polytechnic Institute of Surabaya ${ }^{12}$ \\ Jalan Raya ITS Surabaya 60111, Indonesia \\ E-mail: [civer.yoshioka@gmail.com¹, \{trison,ridho\}@pens.ac.id²]
}

\begin{abstract}
The sense of safety in public facilities for pedestrians can be shown by the availability of good infrastructure, particularly the building. One of the aspects that can make pedestrians feel comfortable and safe is the availability of evacuation facilities in emergency situation. When a disaster strikes, people would start to panic and this will cause problems, especially during an evacuation.During panic in an evacuation process, pedestrians tend to act blindly and walk randomly and mindlessly. They might follow one another when they get panic. This is called as herding behavior. Regarding the evacuation systems, cellular automata is the basic method used to represent human motion. The movement of pedestrian is an important aspect during an evacuation process and this can be analyzed and implemented by using Cellular Automata. It is a simple method yet it can solve complex problems.Total evacuation time becomes the indicators in measuring the efficiency of this system. The result of comparison method shows that the proposed method could work better in certain conditions. In addition, the results of the experiments during panic and normal situation show similar characteristics especially regarding density aspect, yet evacuation time during panic situation takes longer time. The experiment's results by using the actual data also has similar tendency with the evacuation time.
\end{abstract}

Keywords: evacuation time, cellular automata, panic behavior, pedestrian

\section{INTRODUCTION}

Indonesia is one of the countrieswith relatively high frequency of disasters. Every year, the number of the victims of the disaster is quite high. Most of the casualties are caused by the fall of buildings.Many high-rise buildings in Indonesia do not have good evacuation standards. This condition would potentially cause the higher number of victimswhen a disaster occurs. When such disaster occurs, the problem not only lies in whether or not there 
are good evacuation facilities and procedures, but alsoin the individuals themselves.

Pedestrian flow modeling based on cellular automata has been investigated by many researchers in understanding this phenomenon.Cellular automata is a discretemodel suitable for modeling steps or movements of pedestrian. Pedestrian flow modeling consists of three methods: continuum model, social force model, and cellular automata (CA) model. The modeling and analysis of the macroscopic of pedestrian flow uses a continuum model. Three of the macroscopic variables of pedestrian includespeed, density, and flow. Some published papers of the macroscopic variables of pedestrian flow discuss about the continuum model [1][2][3][12]. A concept of continuum model for the flow of pedestrians including the equilibrium system was presented by[1] comparing the stability of disturbances in subcritical flows and supercritical flows, [2]revisiting Hughes's dynamics continuum model for pedestrian flow when it is performed, and[12] developing an efficient algorithm solution. The algorithm used time-varying for the pedestrian generation while the interaction of each variable includes pedestrian density andflux. Walking speed is performed by using conservation equation. An algorithm for an extended reactive dynamic user equilibrium model of pedestrian counterflow as a continuum is developed by [12]. It is based on a cell-centered highresolution finite volume scheme with a fast sweeping method for an Eikonaltype equation on an orthogonal grid. The numerical results demonstrate the rationality of the model and efficiency of the algorithm.

C. Burstedde[1] has developed a model on evacuation process in largeroom to investigate the interaction between pedestrians. In line with that research, this study will simulate pedestrian movement in a building by using similar method with some modifications. This method uses cellular automata asthe basic method for modeling and $\mathrm{v}$ max $=1$ cell ( $v$ max is velocity maximum of pedestrians). According to the research presented by Dirk Heldbing [4], panic pedestrians will run faster than normal pedestrians. Therefore,this study will extend $\mathrm{v} \max =2$ cells to represent the character of panic pedestrians.

\section{RELATED WORKS}

Several methods have been proposed to model pedestrians'behavior. Modeling pedestrians' behaviorsduringlong-range interactions between pedestrian based on Cellular Automataare mediated by floor fields(Cell of Cellular Automata Model) and using chemotaxis approach (C. Burstedde 2001) [1]. The model only simulates pedestrian in a room. Modeling the characteristics of pedestriansduring evacuation process is very complicated. Angsar Kirchner has developed new model in extendingthe model developed by C. Burstedde[1][2]. The model adds some parameter to C. Burstedde's model inanalyzing herding and panic behavior of pedestrians. 
During evacuation process, unexpected things might occur and pedestrians' behavior is unpredictable. In this condition many pedestrians willbump into each other. This study tries to investigate and solve the problem when two or morepedestrians try to enter the same space by using probability as a solution[1][3]. One of the important thingsduring evacuation process is the behavior of pedestrian as affected by panics. Many people will feel scared and urged to find save places while determining what to do. Dirk Helbing[4] has proposed the model to investigatethe pedestrians'behaviorduring such panic and jamming condition.Extended static floor field using djikstra algorithmhas beendone by Katsuhiro Nishinari. His model isimplemented on large room to avoid obstacles [11].

\section{ORIGINALITY}

In this study, we propose a new model approach by modifying the structure of the static floor field[1]. The proposed method is by defining Graph in calculating the distribution value of the static floor field. This study divides the evacuation process into several stages. Evacuation process is divided into two stages: (1) Stage 1: every pedestrian in the room of the building is trying to get out of every room(using previous model); (2) Stage 2: after the people get out of the room, the pedestrians will enter more complex room (corridors of the building). At this stage, new approach will be implemented.

\section{SYSTEM DESIGN}

This study analyzes the behavior of pedestrians in a building when a disaster occurs. When disaster strikes, panic disorder might potentiallybecomea problem in the evacuation process. If pedestrians are inside a building, naturally they willimmediately look for a safe area by using their basic knowledge.This studyapplies model of pedestrian dynamic in large room from previous researchesby adding some modifications. This model uses cellular automata as a basic model for modeling to represent pedestrians. The model combines the Graph to find the shortest distance from the exit of static floor field. This model is expected to provide a solution in this study.

The basic model in this study uses a $3 \times 3$ matrix as neighboring cells. Each matrix is divided into two types. Type 1represents the probability of a pedestrian $\mathrm{s} /$ he doesnot to move to a neighboring cell.Meanwhile, Type 2 has eight-cells to represent the probability of a pedestrian to move to a neighboring cell. One of the neighboringcells will be occupied by pedestrianswhen theymove to the neighboring cell.

\subsection{Floor Field}

Floor fieldin this model consists of two types [1] [2] [3], namely dynamic floor field and static floor field. Dynamic floor field represents the interaction between pedestrians. The valueof dynamic floor field will be 
affected by the movement of the pedestrians when they have moved to a neighboring cell.The value of dynamic floor field occupied by pedestrians will be increased by 1 . Dynamic floor field is influenced by two parameters, namely diffusion and decay. Static floor field has a fixed value. In the previous research, the weight of static floor field is calculated by using an equation to find the shortest distance. When getting closer to the exit, the weight of the static floor field will becomelarger. Static floor field value can be calculated by using the following equation (1):

$$
S_{i j}=\min _{\left(i \tau_{s}, j \tau_{s}\right)}\left\{(i l, j l)\left\{\sqrt{\left(i \tau_{s}-i_{l}\right)^{2}+\left(j \tau_{s}-j\right)^{2}}\right\}-\sqrt{\left(i \tau_{s}-i\right)^{2}+\left(j \tau_{s}-j\right)^{2}}\right\}
$$

The equation(1) was introduced by Angsar K (2002). Equation 1 can be used to calculate the weight of the static floor field, for example, in the case occurring in a large room. That equation is good for modeling case of evacuation in large room where there are no obstacles. Modeling in a large room provides obstaclesthat might make pedestrians stuck on an obstacle. To avoid obstacles, Kanishiro [11] introduced a modification of static floor field by using graph. Graph is made using the corners of obstacleswhich are interconnected with one another. For the proposed model, the graph is defined by the researcher. In this study, the structure of the building is very complicated. It would make the weight distribution becomemore complicated for static floor field.

\subsection{Probability to Move}

C. Burstedde [1] introduced a method in determining the direction of the movement of pedestrians into a neighboring cell by using probability theory.It can be seen on the following equation(2):

$$
\begin{array}{ll}
P_{i j}=N M_{i j} D_{i j} S_{i j}\left(1-n_{i j}\right) \\
\mathrm{P}_{\mathrm{ij}} & : \text { Probability to move } \\
\mathrm{N} & : \text { Normalization factor } \\
\mathrm{M}_{\mathrm{ij}} & : \text { Matrix preferences } \\
\mathrm{D}_{\mathrm{ij}} & : \text { Value of matrix dynamic floor field at index } \mathrm{i}, \mathrm{j} . \\
\mathrm{S}_{\mathrm{ij}} & : \text { Value of matrix static floor field at index } \mathrm{i}, \mathrm{j} . \\
\mathrm{n}_{\mathrm{ij}} & : \text { Indicator of neighboring cell, } 1 \text { if any pedestrian and } 0 \text { if } \\
& \text { empty }
\end{array}
$$

Angsar K. made some modifications to the equations introduced by $\mathrm{C}$ Burstedde. He is more focused on the impact caused by sensitive parameters ( $k s$ and $k d$ ). Both parameters are inserted into the equation(2) to give the effect of herding and blind behavior of the pedestrians. The equation has changed as into the following equation(3). 


$$
P_{i j}=N \exp \left(k_{D} D_{i j}\right) \exp \left(k_{S} S_{i j}\right)\left(1-n_{i j}\right) \varepsilon_{i j}
$$

The $\varepsilon_{i j}$ has the value of 0 if the cell is a wall or obstacle. $K s$ is a sensitive parameter for static floor field and $k d$ is a sensitive parameter for dynamic floor field. This study is intended to see the influence of $k s$ and $k d$ in an evacuation if the experiment is conducted in a building.

\subsection{Solving problem when two or more people enter the same space}

Parallel update allowstwo or more pedestrians went into the same space. This condition would create conflict in the process of updating the rules. When a conflict arises, the equation in Figure 1 and 2 can be used to resolve the problem. In regard to the previous research, the number of neighboring transition matrix was 4 or 8 , but in this case the researcher used8neighboring transition matrices.

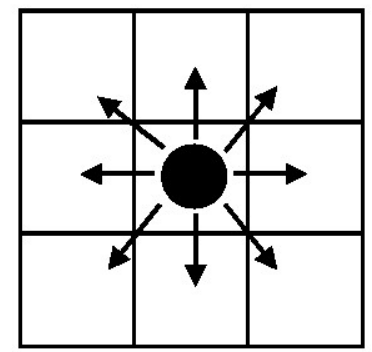

\begin{tabular}{|l|l|l|}
\hline$M_{-1,-1}$ & $M_{-1,0}$ & $M_{-1,1}$ \\
\hline$M_{0,-1}$ & $M_{0,0}$ & $M_{0,1}$ \\
\hline$M_{1,-1}$ & $M_{1,0}$ & $M_{1,1}$ \\
\hline
\end{tabular}

Figure 1. A particle has the possibility to move to the neighboring matrix $\mathrm{M}=\left(\mathrm{M}_{\mathrm{ij}}\right)[1]$.

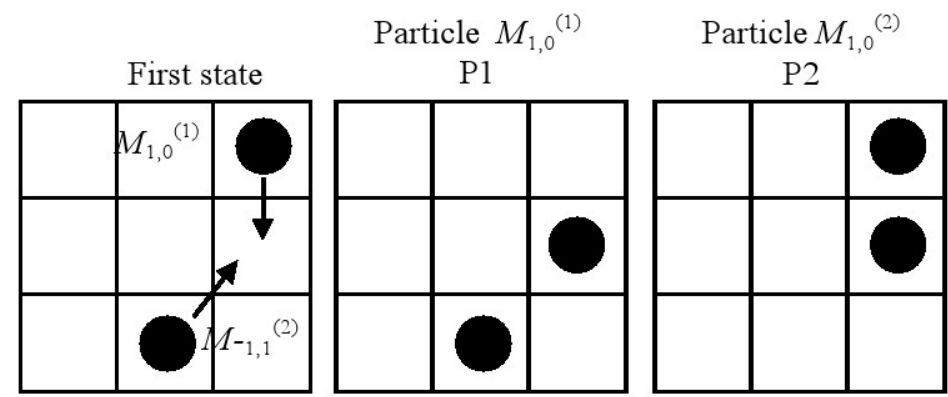

Figure 2. Solution if there are two pedestrians (M (1) and $\mathrm{M}^{(2)}$ ) moving to the same neighbor cell.

$$
\begin{aligned}
& \mathrm{P} 1=\frac{M 1,0(1)}{M 1,0(1)+M 1,0(2)} \\
& \mathrm{P} 2=\frac{M 1,0(2)}{M 1,0(1)+M 1,0(2)}
\end{aligned}
$$

The above conditions areused when there are two or more pedestrians moving into the same cell in one time space. 


\subsection{Update Rule}

In applying this method, some rules such as the ones used in the previousstudies were used [1] [2] [3]. The following rules are applied to each pedestrian based on parallel update in discrete time step $(t \rightarrow t+1)$ :

1. Dynamic floor field controlled by diffusion and decay processes.

2. Each pedestrian has a transition probability to move to another neighbor cellwhich is determined by Equation ${ }^{(2)}$ and will be influenced by two sensitive parameters $k s \in[0, \infty]$ and $k d \in[0, \infty]$.

3. Every pedestrian will move to a neighboring cell based on the transition probability of the previous step.

4. If there are two or more pedestrians who chose the same cell targets, this problem will be solvedby the above method(2.1).

5. Dynamic floor field value will increase by the movement of pedestrians $(\mathrm{D} \rightarrow \mathrm{D}+1)$.

The rules above are used to update the movementof all pedestrians at the same time space in parallel update.

\subsection{Panic Behavior}

One of the interesting things in evacuation process is the sense of panic experienced by the individual.This panic phenomenon during the occurrence of disaster has some effectstowards the evacuation and it makes the evacuation process become more complicated. In the process of evacuation, panic behavior often leads to death.Their panic might cause them to stumble, fall or get trampled by others [4]. This study tries to insert some of the characteristics of panic behavior into the simulation. The previous studies have described some of the characteristics of panic behavior when a disaster strikes. This experiment does not use all the characteristics of panic behavior. This experimentuses some limitations by using only a few panic characteristics to this simulation, which are:

- Getting nervous and acting blindly.

- Running faster than normal pedestrian.

- Showingherdingbehavior, which is byfollowing each other, especially the larger group.

- Pushing each other at any exits while alternative exits are often not efficient for them to escape.

The research wasconducted by Angshar K (2002).The parameter ks and $k d$ have some effectsin representing the panic behaviors. The lower the value of ksmeans the higher the chance of pedestrians acting blindlyin looking for the exit and it means they will show more random behaviors, such as running mindlessly. The higher value of $k s$ means that the pedestrians know better about the exit position and the surrounding 
environment. The higher the value of $k d$ means that the pedestrians showsherding behavior. To represent the behavior of panic that has been described previously, this study uses the combination of $k d$ and $k s$ values.

\subsection{Proposed Concept}

In Computer science, graph is defined as a structure used to model pairwise relations between objects. The interconnected objects are represented by mathematical abstractions called vertices, and the links that connect some pairs of vertices are called edges. Graph $(G=<V, E>)$ consists of unit of vertices $(V)$ and unit of edges $(E)$. Edge formulation $(E=(u, v))$ means thatEdge (e)is connected by two points, which are $u$ and $v$. To make the computation easier, this study uses directed graphs.

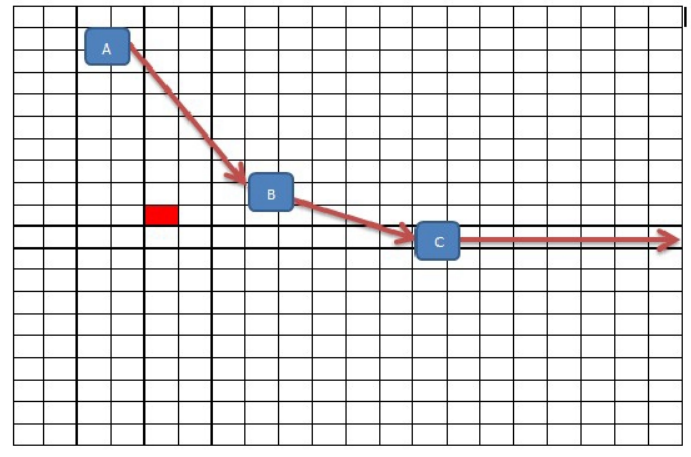

(a)

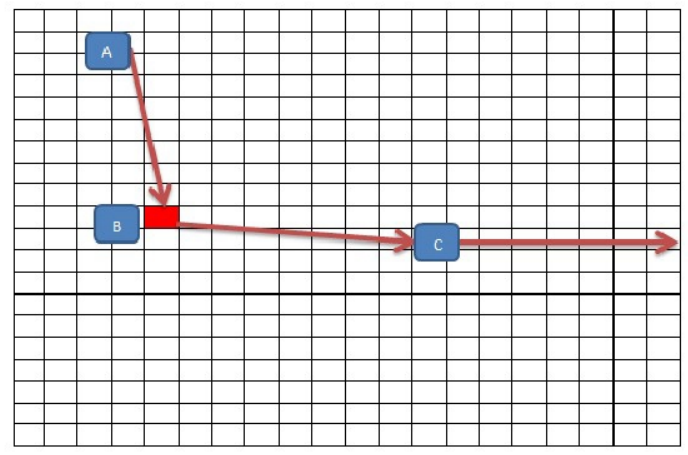

(b)

Figure 3. (a) Initial of cell, (b) Calculate value of cell

Figure 3 describes how the graph is used to calculate the distance to the nearest exit. In the previous study [2], the simulation is performed only in a room.To calculate the value of static floor field, the researcher used Euclidean equation to find the shortest distance to the exit. Steps for calculating the value of static floor field of the proposed method is described as follows:

1. Definingthe position of Matrix $\left(\mathrm{M}_{i, j}\right)$ of the sequence of the cell. Figure 5 is illustrated in the red cell.

2. Findingthe nearest vertices or nodes, which-in the example above-is vertices $B$.

3. Replacingthe nearest position of vertices $\left(\mathrm{V}_{i}\right)$ with the position of matrix $\left(\mathrm{M}_{i, j}\right)$ cell.

4. Calculating new edge distance of replaced vertices.

5. Calculating total distance of the nearest exit.

6. Repeating steps 1-5 until the last cell.

Weight edge $E=(u, v)$ is calculated by using Equation 4 . 


$$
W E_{i}=\sqrt{\left(u_{i}-v_{i}\right)^{2}+\left(u_{j}-v_{j}\right)^{2}}
$$

In which :

$$
\begin{array}{ll}
W E_{i} & =\text { Weight of egde } i . \\
u_{i} \text { and } u_{j} & =\text { Position of the first vertices }(V) \text { on Matrix }\left(M_{i j} .\right) \\
v_{i} \text { and } v_{j} & =\text { Position of the second vertices }(V) \text { on Matrix }\left(M_{i j} .\right)
\end{array}
$$

When the value of $W E$ is already found, the nearest Vertices $\left(V_{i}\right)$ to cell of Matrix $\left(M_{i j}\right)$ is computed by using Equation 5.

$$
N V=\min \left\{\sqrt{\left(x_{i}-x v_{i}\right)^{2}+\left(y_{j}-y v_{j}\right)^{2}}\right\}
$$

In which :

NV $\quad=$ Nearest weight of new Vertices.

$x_{i}$ and $y_{j} \quad=$ Position of the cell on Matrix $M_{i j}$.

$x v_{i}$ and $y v_{j}=$ Position of the vertices $V_{i}$ on Matrix $M_{i j}$.

$N V$ is the closest distance value between matrix $\mathrm{M}_{i j}$ and Vertices $\mathrm{V}_{i}$. We can calculate the value of all the edges connected with Vertices $V_{i}$, and then calculate all the possible paths toward an exit and find the closest value with Equation 6.

$$
T W_{i j}=\min \left\{N V+\sum_{i=0}^{n} W E_{i}\right\}
$$

$T W_{i j}$ is the total value of distance Matrix $\left(M_{i j}\right)$ to the nearest exit door. Equation 5 and 6are implemented on all iterationswhile Equation 4is only implemented once, at the first time when running the application.

\subsection{Map in Case Study}

This research combines the proposed methods and the previous methods for evacuation in a building. The location chosen as the model to conduct this virtual experiment was Marina Plaza, Surabaya, Indonesia considering that Marina Plaza is one of the shopping malls in Surabaya which has relativelyfrequent number of visitors. The detailsof the view and map of Plaza Marina Surabaya can be seen in Figure 4. 


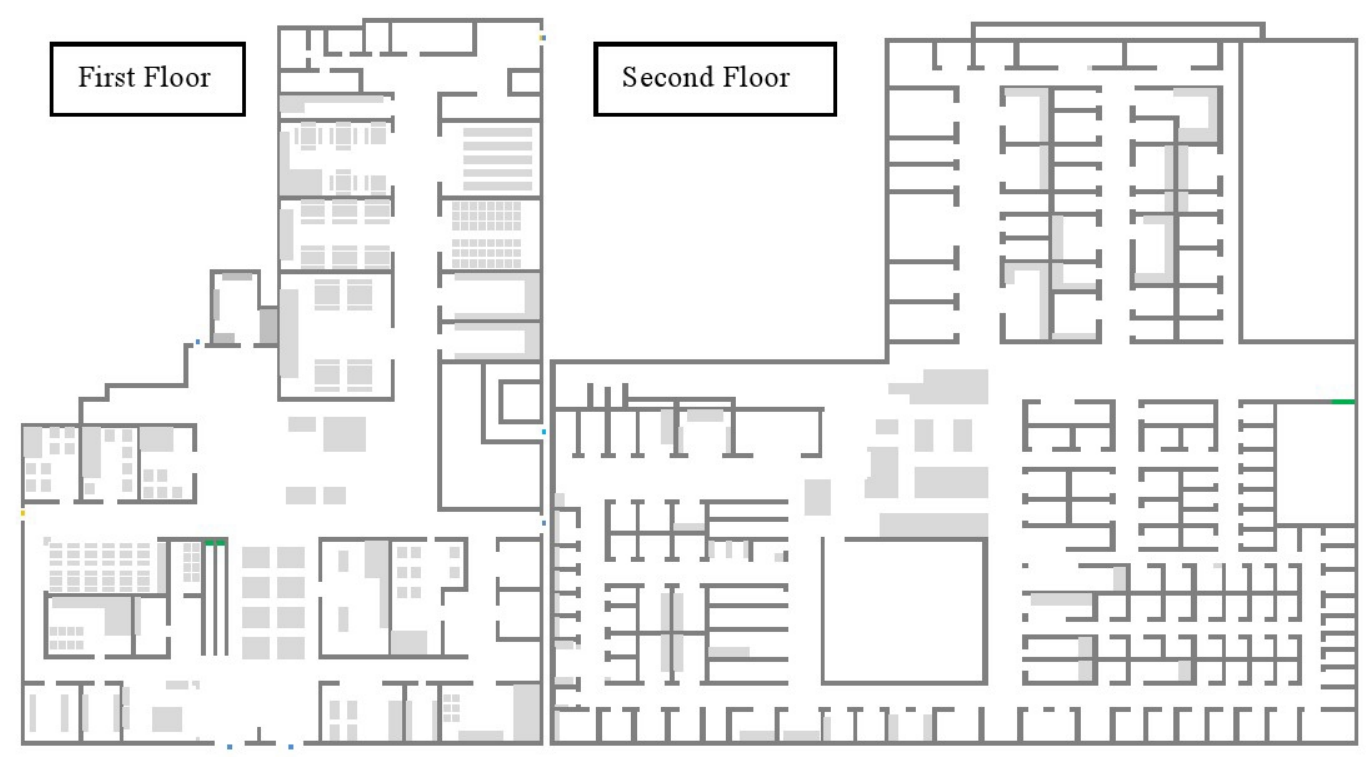

Figure 4. Map of Marina Plaza Surabaya

\section{EXPERIMENT AND ANALYSIS}

This section will discuss the impact of sensitive parameters ( $k s$ and $k d$ ) that are inserted into this simulation. This experiment is divided into three parts: Comparison between the Previous and the Proposed Methods Related to Static Floor Field, Simulation of Evacuation based on the Panic and Normal Characters, and Simulation of Evacuation using Actual Data.

\subsection{Comparison between the Previous and the Proposed Methods Related to Static Floor Field}

To see the performance of the proposed method, this section will compare the previous method[1] and the proposed method. Virtual experiment was conductedby making simulation in large rooms with the same specifications by using some obstacles as seen in the maps below:

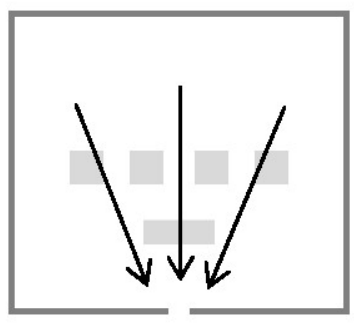

(a)

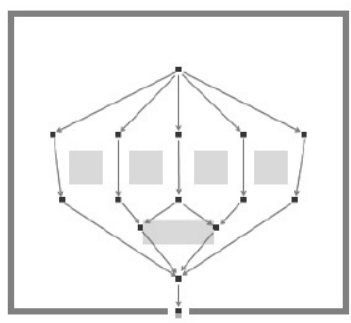

(b)

Figure 5. Two maps(a) and (b) to compare the previous and the proposed method. 
Figure 5 shows two mapsused for comparison between the previous and proposed method. The specificationsof the room can be seenin the list below:

1. Size of the room is $73 \times 65$ cells and the room has one door.

2. Size of the door is 5 cells.

3. The area of each cell is $40 \times 40 \mathrm{~cm}^{2}$

The proposed method to solve the problem when there is any obstacle in front of door can be seen in the Figure 6 and 7 below.

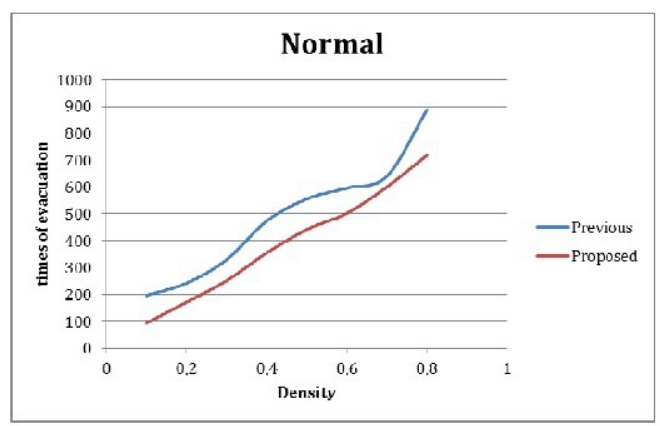

(a)

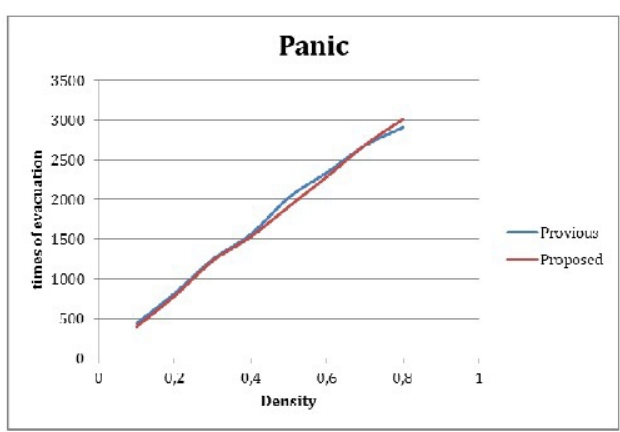

(b)

Figure 6. Relational graphics of evacuation time and density of both normal and panic pedestrians.

According tothe relational graphics, simulation modeling in normal conditions is shown in Figure 6 (a). The evacuation time difference between the previous and the proposed method is quite high because in this situation the pedestrianshave a good knowledge to find the exit. This condition makes the view of pedestriansis only focused toward the exit. This condition makes themget stuck on obstacles and it becomes difficult for them to find the exitif there are obstacles in front of the door. In Figure 6 (b), the evacuation time difference between the previous and the proposed method is quite low.Pedestrians in panic situation tend to be nervous and act blindly. This condition makesthem walk or run randomly and mindlessly. In this condition, there would be lowertendency to get stuck on an obstacle. As a consequence,the evacuation time lap between the two methods islow, yetthe evacuation time needed in panic situationhighly increases. 


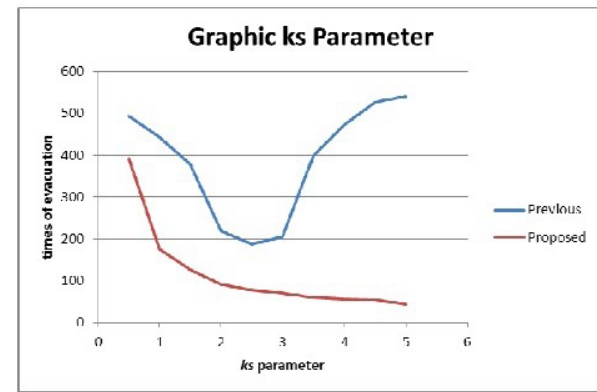

(a)

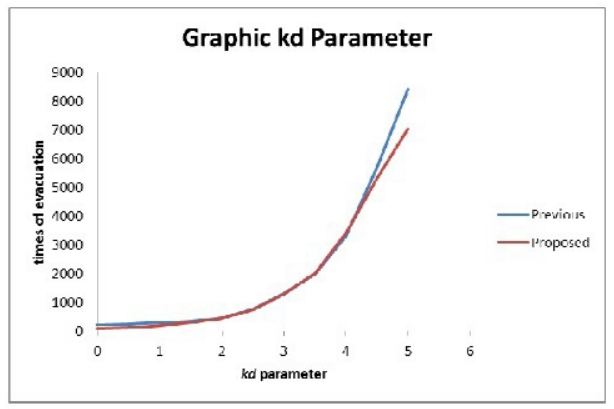

(b)

Figure 7. Relational graphics of evacuation time, $k d$ and $k s$.

Ks parameter representsthe pedestrians' knowledgeon the location of the exit. Adding obstacles in the large room simulation will result in higherevacuation time in certain conditions given that some pedestriansare trapped by obstacles and it becomes difficult for them to find the exit. In Figure 7 (a), the evacuation time does not decrease regularly.When the value of $k s=3$, evacuation time increases. In this condition, evacuation time is supposed to decreases regularly[1]. The higher value of $k d$, pedestrians will follow each other(herding behaviors) and act blindly. In this condition, the possibility of the pedestrian to get trapped becomes smaller,yet the evacuation time will consequently increase. InFigure 7 (b), the time difference evacuation between the previous method and the proposed method is quite small.

\subsection{Simulation of Evacuation based on the Panic and Normal Characters}

In the previous studies, the effect of $k s$ and $k d$ in the large room was studied by Angsar K. If $k s$ is lower than 1, it means that the pedestrians do not have enough knowledge about the environment. It suggests that the pedestrians are confused to find the exit and assumethat the room is full of smoke, in case of fire. If $k s$ parameter is bigger than 1, it means the pedestrianshaveenough knowledge about the environment and know the location of exit. Meanwhile, $k d$ parameterhas different influences. If $k d$ is higher than 1.Kd parameter has the effect of making the pedestrians lead and follow each other. In this experiment, the characteristic of pedestrians has been divided into two characters by using the combination of $k s$ and $k d$. This combination is made to divide the characteristics of the pedestrians when they are in normal situation and when they are in panic. For more details, it can be seen below:

Panic Character $\quad: k s$ parameter is 1 and $k d$ parameter is 2

Normal Character $\quad: k s$ parameter is 2 and $k d$ parameter is 1

The above combinationis one of the examples ofcombinations that would be modelled. The value range of $k s$ dan $k d$ in the previousresearch is 0 
toinfinite. However, the researcher will still refer to the previous statement on howthe impact would be if the kdandks value is higher. Hence thecombination of $k s$ and $k d$ such as above is chosen to representthe character of the pedestrians in panic and normal situation.

The simulations have been done by using real data from Marina Plaza Surabaya on the first and the second floors. The second floor is one of the most crowded areas. Evacuation route in this building has already been determined by MarinaPlaza Surabaya.This experiment will be based on the data of the evacuation route of MarinaPlaza Surabaya. The specifications of the simulation can be seen below:

1. The area of each cell is $40 \times 40 \mathrm{~cm}^{2}$

2. The number of exits is six doors.

3. The size of the building is $179 \times 217$ cells and the experiment only usesthe first and the second floors.

4. The case study is situated at one of the shopping centers in Surabaya.

5. The plaza building has 3 floors but this case studytakes place on the first and the second floors.

6. The evacuation route is based on the emergency evacuation route of Marina Plaza Surabaya.

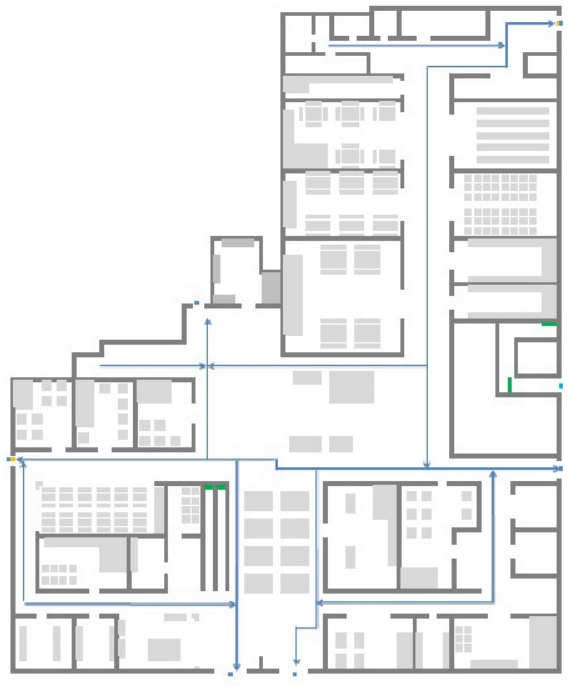

(a)

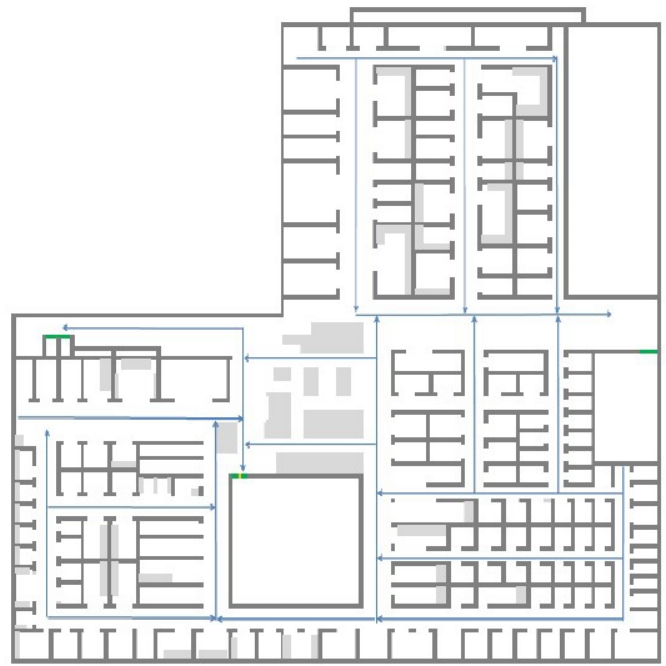

(b)

Figure 8. (a) Route of evacuation on the first floor, (b) Route of evacuation on the second floor

This experiment is based on the evacuation route of Marina Plaza Surabaya. The meaning of "evacuation route" is the evacuation route used by Marina Plaza Surabaya when disaster occurs. In general, all the buildings have emergency evacuation standards, but every building management should have emergency evacuation standards adapted to their building 
standards. This study has followed the routes of evacuation at Marina Plaza Surabaya. Details of the evacuation route can be seen in Figure 8. Figure 8(a) is the first floor and Figure 8(b) is the second floor.

This simulation uses two above characters: in panic and normal situation. These characters have been inserted into the modeling. For more detailsof the result of the experiment, see Figure 9 below.

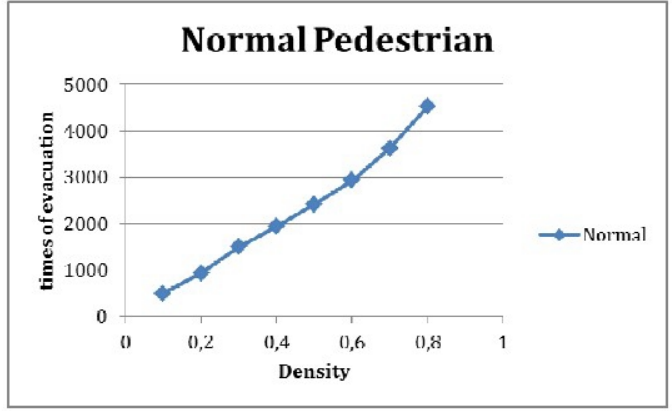

(a)

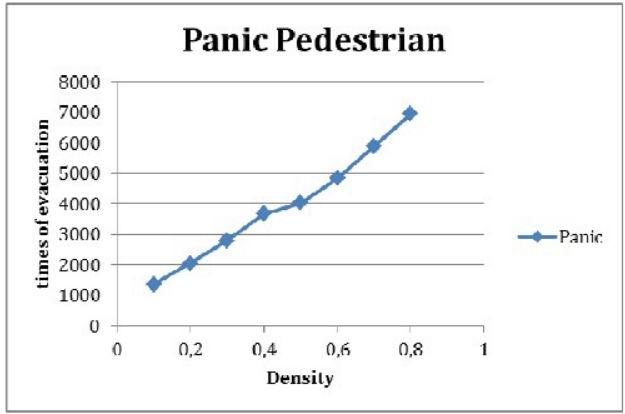

(b)

Figure 9. Relational graphics of evacuation time and density proposed method.

Figure 9 (a) is an experiment to findtherelation betweentime of evacuation and density ofnormal characteristics. Basically, greater value of density will be followed by increased evacuation time. Figure 9 (b) is an experiment to find therelation between time of evacuation and density for pedestrians with panic character. In this experiment, greater value of density will be followed by increased evacuation time. When looking into the two graphics above it can be concluded that the two graphics above have the same characteristics, but they have different value of evacuation time.

\subsection{Simulation of Evacuation using Actual Data}

This section is intended to determine the evacuation time by using actual data. Shopping centers,particularly Marina Plaza Surabaya, has a different number of visitors duringnormal (weekdays) and weekends/public holidays. Surabaya Local Government has divided shopping center activities into three parts: low density(visitors on Monday, Tuesday, and Wednesday),middle density(visitorson Thursday and Friday), andhigh density(visitorson Saturday and Sunday(weekend)).

Table 1. Number of Visitors on one Week

\begin{tabular}{|l|l|c|}
\hline Num & Category & Number of Visitors \\
\hline 1 & Low & $929 /$ hour \\
\hline 2 & Middle & $933 /$ hour \\
\hline 3 & High & $1164 /$ hour \\
\hline
\end{tabular}


Table 1 is the estimation of the number of visitors at Marina Plaza Surabaya in one week. The number of visitors is calculated by the number of cars and bicycles parked at Marina Plaza Surabaya's parking lot. The number of visitors used is only that in the first and second floors. The most crowded floor is the second floor because the second floor is the center of Marina Plaza Surabaya. According to Table 1,the difference between the number of visitors in the category of Low and Middleare not high. The number of visitors in Table 1 is the average number of visitors per hour. To see the results of the experiment, we can see the graphics below.

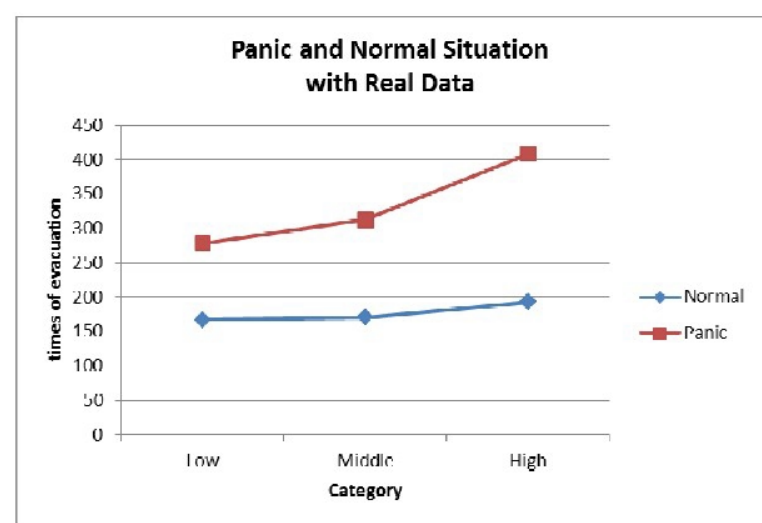

Figure 10. Relational graphics between Panic and Normal Pedestrians usingActual Data.

According to the graphic in Figure 10, the evacuation time in Low and Middle category have small difference. The characteristics number of visitors of Marina Plaza Surabaya on Monday to Friday does not show high increase. However,during weekend, the number of visitors has increased quite a lot. This condition makesthe evacuation time falls to the category of High because the time increases quite a lot (see Figure 10).The experimental results by using the actual data showthe same tendency of evacuation time, yet the panic character has higher evacuation time than normal characters.

\section{CONCLUSION}

Basic model proposed by C. Burstedde can be implemented to modeling evacuation process in abuilding by using some modifications of static floor field. This study modifies the distribution of static floor fieldfrom the previous study so the proposed method can be applied in a building. The experiment shows that the proposed method works better than the previous method when applied in certain condition.The calculation of the distribution value of static floor field can be implemented by using Graph. Graph is used to give direction to pedestrians to follow the evacuation route in a building, in this case Marina Plaza Surabaya. According the expperiment results, it can be concluded that defining the direction of Graphsis very important in determining the duration of evacuation time. By using the modification of static floor field, it shows thatproposed method can be implemented on 
evacuation process in abuilding.This model is expected to help analyze the panic behavior of pedestrians when disaster occurs, particularlyat Marina Plaza Surabaya.By implement this method, it is hoped that in the future management of buildings for public facility could design much better system of evacuation standards especially for the safety of the visitors.

\section{REFERENCES}

[1] Burstedde C, Klauck K. Simulation of Pedestrian Dynamics Using a Twodimensional Cellular Automaton, Institut fur Theoretische Physik, Germany, 2001. Physica A 295 (2001) 507-525.

[2] Kircher A, Scadscheneider A.Simulation of Evacuation Process Using a Bionics-inspired Cellular Automaton Model for Pedestrian Dynamics. Koln University, Germany, Physica A 312 (2002) 260 - 276

[3] Kircher A, Katsuhiro N.Friction Effect and Clogging in a Cellular Automaton Model for Pedestrian Dynamics. Koln University, Germany, 2008.

[4] D. Helbing, A. Jhohansson. Pedestrian, Crowd and Evacuation, ETH Zurich, Zurich, Switzerland.

[5] Dos Santos Robson F, Das Gracas Maria B, Born Margarethe S., Simulating Collective Behavior in Natural Disaster Situations: A Multi Agent Aproach, Brazil, 2012.

[6] Cristian Pablo T, Printista marcela, Luis Marcelo E.Evacuation Simulation using Cellular Automata, San Luis National University of Argentina, 2007. JCS\&T Vol. 7 No. 1.

[7] Nan GAO, Wengguo Weng, Wei MA, Shunjiang NI, Quanyi HUANG, Honhyong YUAN, Fire Spread Model for Old Towns Based on Cellular Automaton, Cina, 2008. Tsinghua Science and Technology,October 2008, 13(5): 736-740.

[8] Vorst Harrie C.M.,Evacuation Models and Disaster Psychology, University of Amsterdam, 2010. H.C.M. Vorst / Procedia Engineering 3 (2010) 1521.

[9] Murphy, Brown K, Sreenan C, Problem Decomposition for Evacuation Simulation using Network Flow. Department of Computer Science, University Col-lege Cork, Ireland, 2012 IEEE/ACM.

[10] N Christian, Cellular Automata Modeling for Pedestrian Dynamis,Mathematicsh Naturwissenschaftliche Fakultat, Universitat Greifswald, Bachelor Thesis.

[11] Nishinari Katsuhiro, Extend Floor Field CA Model for Pedestrian Dynamics, Department of Applied Mathematics and Informatics, Ryuko University, Shiga, Japan.

[12] Harsono Tri, Human Behavior Based Evacuation in A Large Room UsingCellular Automata Model For Pedestrian Dynamics, KCIC 2014, Malang Indonesia.. 